\title{
STIMULATION OF MYENTERIC PLEXUS NEURITE OUTGROWTH BY INSULIN AND INSULIN-LIKE GROWTH FACTORS I AND II
}

\author{
Michael W. Mulholland, Gail Romanchuk, \\ Diane M. Simeone, Kenneth Flowe \\ Michigan Gastrointestinal Peptide Center, 2922 D Taubman, \\ University of Michigan, Ann Arbor, Michigan 48109-0331 \\ (Received in final form september 28, 1992)

\section{Summany}

A defined culture medium containing insulin, insulin-like growth factor 1 (IGF-I) or insulin-like growth factor II (IGF-II) supported morphological development of myenteric plexus neurons derived from neonatal guinea pigs. Insulin increased neurite outgrowth 3-fold at concentrations as low as $0.2 \mathrm{nM}$. Similar significant and dose-dependent increases in neurite outgrowth were noted with IGF-I and IGF-II. Stimulation of neurite outgrowth was abolished by exposure to cytosine arabinofuranoside, an agent toxic to non-neuronal celis, implying that trophic effects of insulin or insulin-like growth factors require the presence of non-neuronal elements in culture.

While nerve growth factor is widely recognized as a physiologically important peptide capable of stimulating neurite outgrowth, recent evidence indicates that insulin and insulin-like growth factors may also have neurotrophic properties. Specific receptors for insulin and the structurally related peptides, insulin-like growth factors I and II (IGF-I and IGF-II) have been identified in central nervous system tissues of a number of species $(3,5,17,19)$. Specific receptors have also been identified in cell lines derived from rat neuroblastoma and pituitary tumor cells $(15,18)$.

Although the functional significance of these observations is not clear, one possibility is that insulin and its homologues play a role in nervous system development. Consistent with this possibility, insulin and IGF-I have been shown to support brain neuronal survival in cell culture $(1,2)$. In fetal rat brain cultures, IGF-I stimulates DNA synthesis (8). Insulin has been noted to stimulate cholinergic development, as assessed by choline acetyltransferase activity, in cultured chicken retina neurons (7). Trophic effects may exist for peripheral nervous system tissues as well; insulin and IGF-II promote neurite formation and survival in cultured sympathetic and sensory neurons (14).

The actions of insulin and insulin-like growth factors on enteric neurons have not been reported. In this study, cultured myenteric plexus neurons derived from neonatal guinea pigs were used to investigate potential trophic activities of insulin, IGF-I, and IGF-II. Studies utilizing enteric neurons in primary culture have several advantages in this regard: 1) measurements can be made without interference from gut epithelial cells, smooth muscle, and endothelium; 2) reliable delivery of known 
concentrations of peptides to target neurons can be assured; and 3) enteric plexus neurons can be observed individually for assessment of morphologic development. In the current study, the experimental goals were three-fold: 1) to assess the ability of insulin, IGF-I and IGF-II to stimulate neurite outgrowth in myenteric neurons; 2) to determine the time course of trophic actions; and 3) to investigate whether these actions are exerted directly upon enteric neurons or require the presence of nonneuronal cells within the culture system.

\section{Methods}

The culture method used has been described previously (6). Two day old male guinea pigs were used. Using aseptic technique, the colon was exposed and the longitudinal smooth muscle of the taenia coli was dissected from the cecum. Care was taken not to enter the lumen of the bowel. The taenia coli was divided into segments and transferred to a Petri dish containing Hank's balanced salt solution (HBSS) with 1 $\mathrm{mg} / \mathrm{ml}$ highly purified collagenase. The muscle segments were incubated at $4^{\circ} \mathrm{C}$ for 12 hours and for 30 to 60 minutes at $37{ }^{\circ} \mathrm{C}$. Tissue fragments were then transferred to fresh HBSS without collagenase, and using a dissecting microscope, loosely adherent smooth muscle fibers were removed from the myenteric plexus.

The tissue derived from each experimental animal was divided into 12 aggregates. The plexuses were then placed into tissue culture wells that had been coated with poly-L-lysine $(10 \mathrm{ug} / \mathrm{ml})$. The explants were exposed to a defined culture medium in the presence or absence of various concentrations of insulin, IGF-I, or IGFII. The medium was composed of serum-free medium 199 supplemented with nonessential amino acids $(0.1 \mathrm{mM})$, sodium pyruvate $(1 \mathrm{mM})$, HEPES buffer $(1 \mathrm{mM})$, Lglutamine $(2 \mathrm{mM})$, glucose $(5 \mathrm{mg} / \mathrm{ml})$, and penicillin-streptomycin solution $(100 \mathrm{U} / \mathrm{ml})$. Culture medium was changed every second day; antibiotics were omitted after the second day. In order to determine if trophic effects were exerted directly upon enteric neurons, some cultures were exposed to cytosine arabinofuranoside (ARA-C: 2.5 $\mathrm{mg} / \mathrm{ml}$ ), an agent cytotoxic for non-neuronal cells but not for neurons (16).

Cultures were examined using phase contrast microscopy. A visual record was maintained using photomicroscopy, and quantitative image analysis was performed daily. Neurite outgrowth was assessed in terms of maximum neurite extension (mean neurite length) and number of newly-formed neurite processes (neurite desity). Plexuses were scored for length of neurite outgrowth by measuring the 5 longest individual neuritic processes per plexus. Neurite length was expressed as the mean of these five measurements. Neurite density was expressed as the number of individual neurite processes extending circumferentially from each plexus. The individual performing the morphometric analysis was unaware of the experimental conditions to which the cells had been exposed. To assess inter-assay variability, measurements were repeated at monthly intervals. Variability was less than $10 \%$ in each instance.

All results are expressed as group means \pm SEM. Each experimental condition was examined using the tissues of 6 to 8 animals. Sample size $(n=6$ or 8$)$ was taken as the number of animals. Statistical analysis was performed using analysis of variance, with post hoc testing performed when significant differences were noted. Significance was accepted as $p<0.05$.

\section{Results}

A defined culture medium, containing insulin, IGF-I or IGF-II supported the morphological development of myenteric plexus neurons derived from neonatal 
guinea pigs. A characteristic pattern of neurite outgrowth was observed, beginning with an zone of non-neuronal cells surrounding the plexus, followed by radial extension of neurites from the plexus, and progressive elongation of neuritic processes (Figure 1).
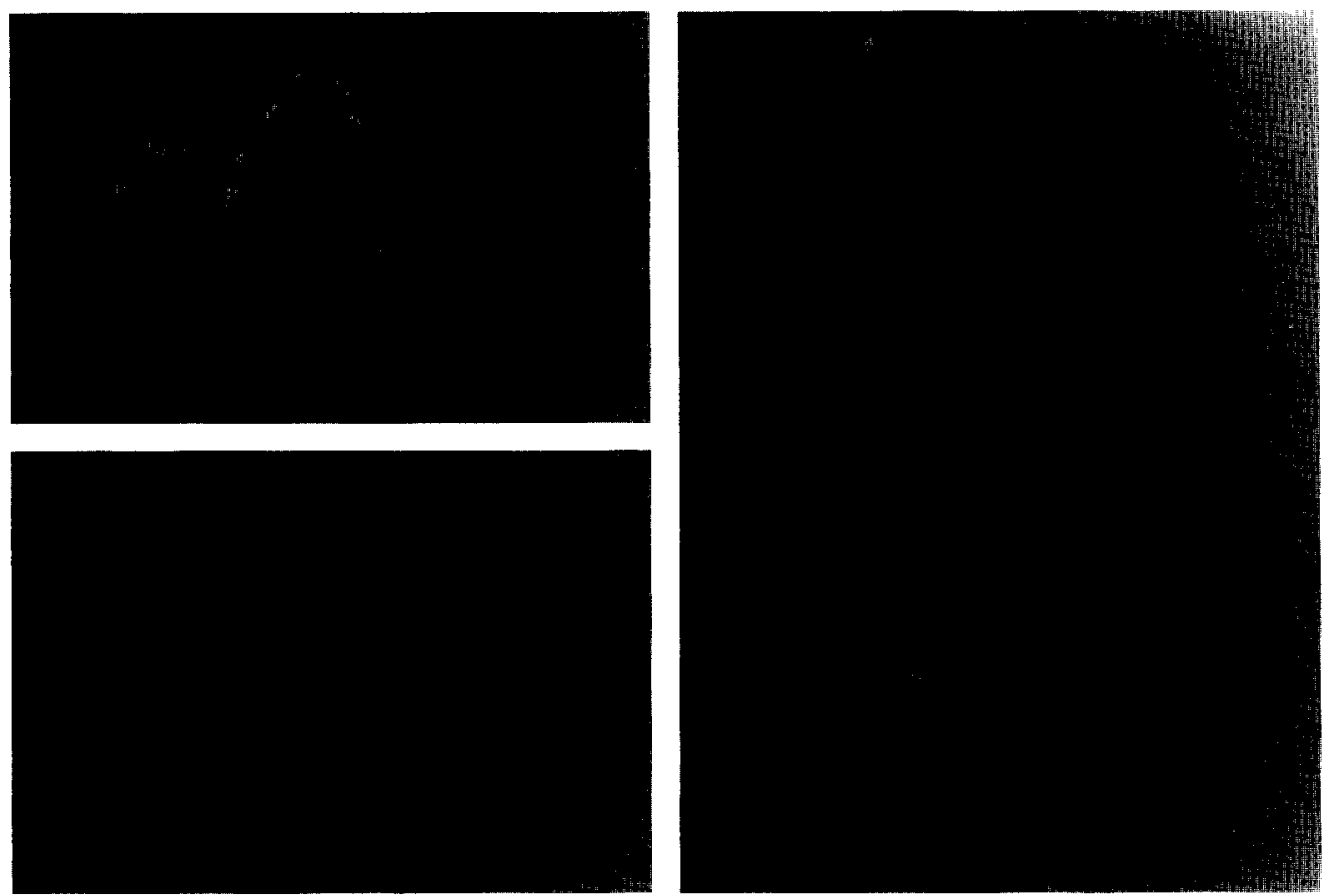

FIG 1

Progressive neurite outgrowth (arrows) in myenteric plexus explant exposed to $5 \mathrm{nM}$ IGF I for $24 \mathrm{~h}$ (A), $72 \mathrm{~h}(\mathrm{~B})$, or $120 \mathrm{~h}$ (C) (100X magnification).

The effects of insulin in serum-free medium upon neurite density and length is shown in Figure 2. Insulin significantly increased both neurite density and neurite length at each concentration tested, with a greater than 3-fold increase in length measurements relative to control noted at a concentration of $0.2 \mathrm{nM}$. Significant differences in neuritic density and length were observed as early as 48 hours and persisted during the remainder of the experiment. 
IGF-I also produced a significant and sustained increase in neurite density and length in cultured neonatal enteric neurons (Figure 3). Stimulation of neurite outgrowth was significantly greater than control at time points from 48 to 120 hours.
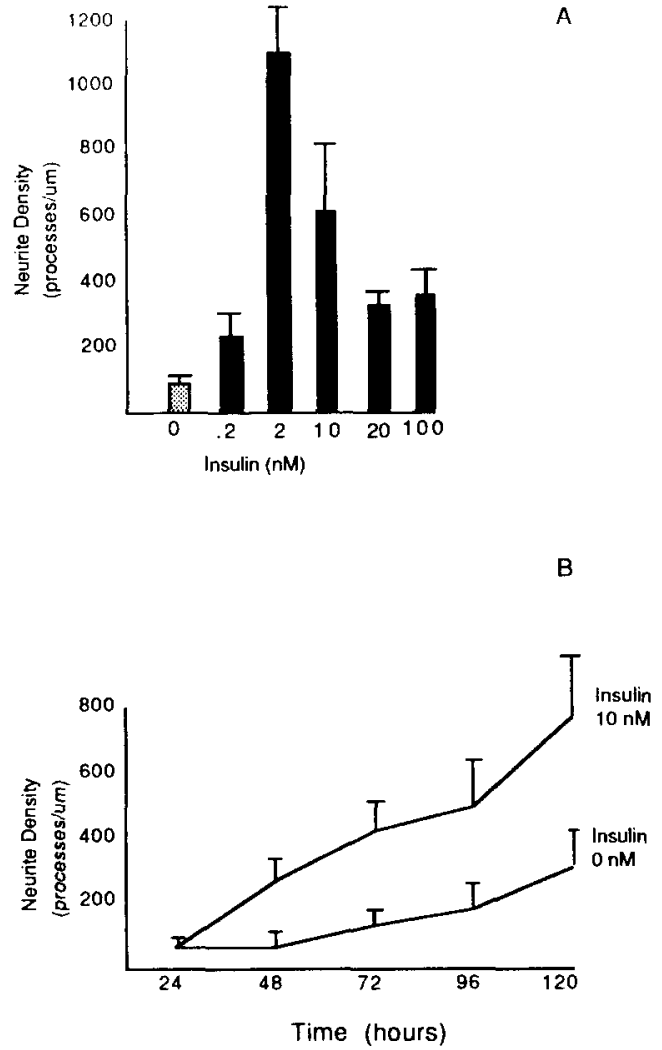

B
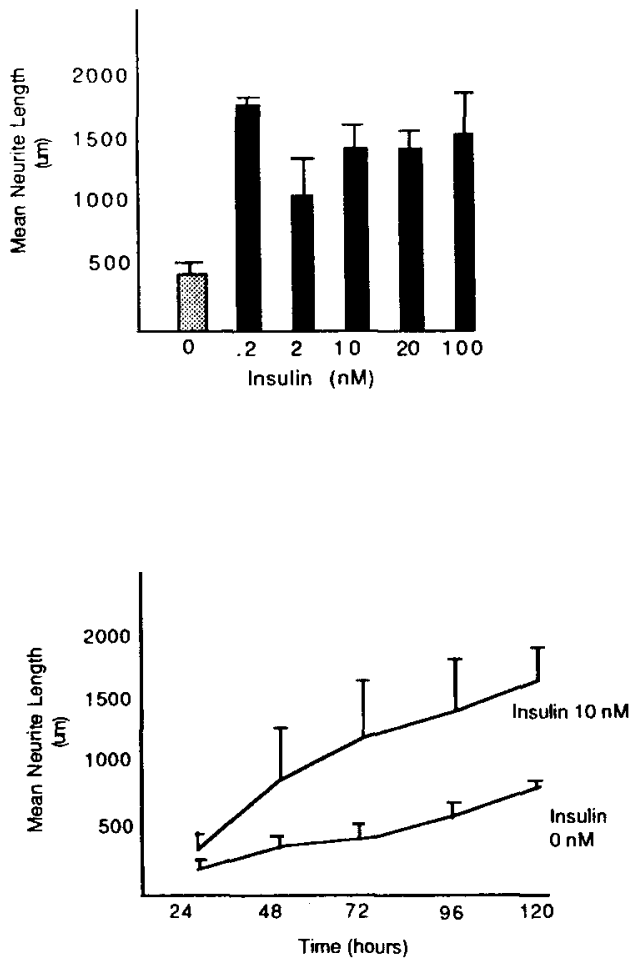

FIG 2

Figure 2. Neurite outgrowth, expressed as neurite density and mean neurite length, in response to graded concentrations of insulin. All values at concentrations of $.2 \mathrm{nM}$ or greater (A) and at intervals of 48 hours or greater $(B)$ were significantly increased relative to control.

IGF-II also supported the morphological development of enteric neurons in primary culture (Figure 4). Increases in density were noted at peptide concentrations as low as $0.1 \mathrm{nM}$. At $10 \mathrm{nM}$ IGF-II, a 2.5 -fold increase in neurite length relative to control was observed. Increases in density were significant by 48 hours and were sustained throughout the study period.

In a separate group of experiments, plexuses were exposed to insulin (2nM), IGF I $(5 \mathrm{nM})$, or IGF $\|(5 \mathrm{nM})$ in the presence or absence of ARA-C. Exposure to ARA-C 
completely abolished trophic activity of insulin (control $353 \pm 43$, insulin $686 \pm 85$, insulin + ARA-C $341 \pm 81$ um mean neurite length) and IGF II (IGF I| $583 \pm 27$, IGF II + ARA-C $354 \pm 59$ ), and significantly blunted trophic actions of IGF I and II, as expressed by increases in neurite length (Figure 5 ).
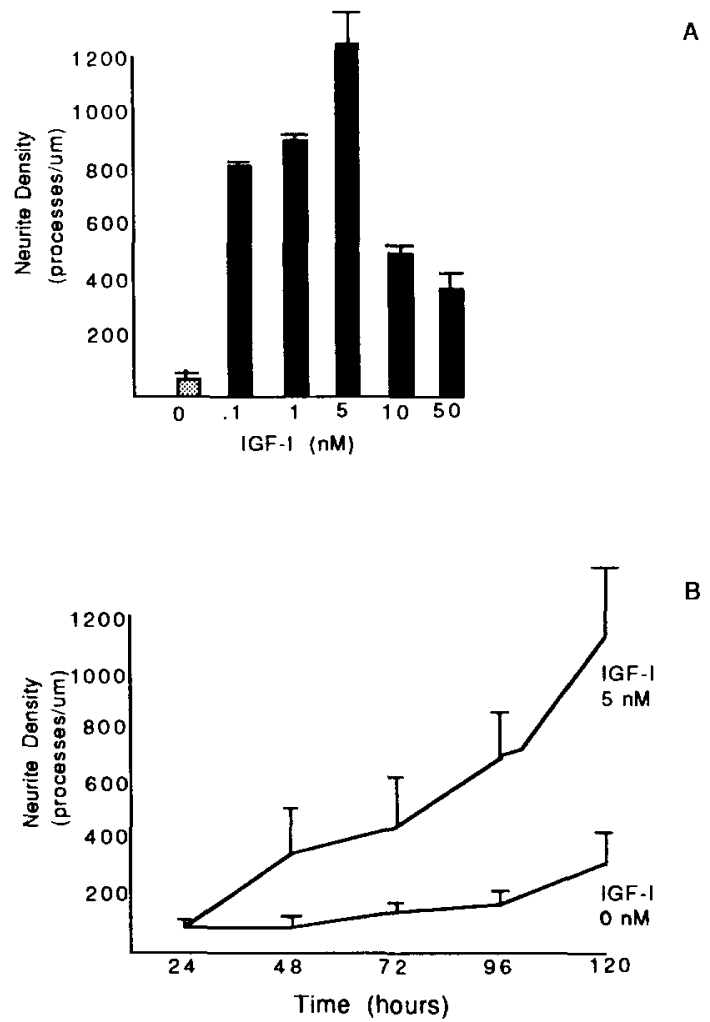

A

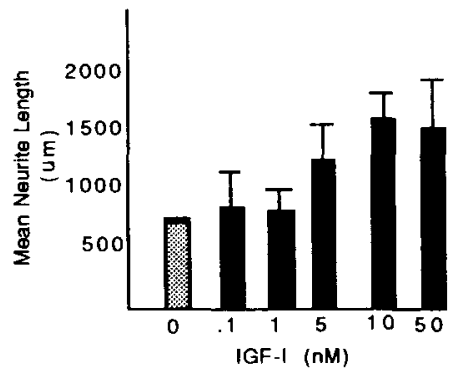

B

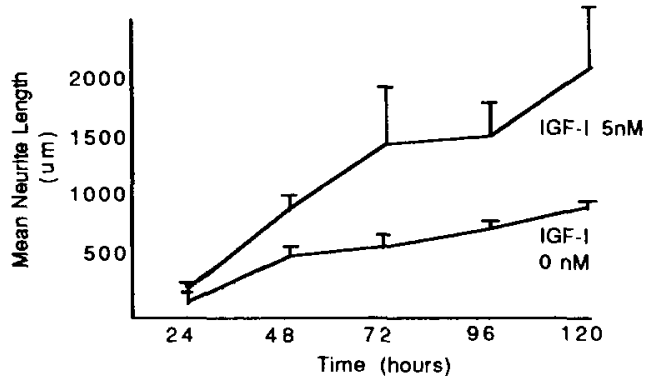

FIG 3

Neurite extension in response to graded concentrations of IGF I. Significant increases were noted for values of $0.1 \mathrm{nM}$ or greater (neurite density) and $5 \mathrm{nM}$ or greater (mean neurite length: panels $A$ ) for all time intervals of 48 hours or greater (B).

\section{Discussion}

The results of the current study indicate that insulin, IGF-I and IGF-II can enhance enteric neuronal outgrowth. Three observations support this contention: 1) for all three peptides, significant increases in neurite density and length, reflecting elongation of newly-formed neurites, were observed; 2) significant effects were noted with peptide concentrations in the nanomolar range; 3) trophic effects were noted as early as $\mathbf{4 8}$ hours in culture and persisted throughout the experimental period. Insulin, IGF-I, and IGF-II appear to require the presence of non-neuronal cells in the culture

It is important to note that enteric neurons survived up to 120 hours in the defined, serum-free medium used in control experiments. Further, small increases in neurite density and mean neurite length were observed in control groups exposed to a serum-free medium, perhaps reflecting low-level intrinsic neurotrophic activity expressed within the plexuses. It is possible that the stimulatory action of insulin, IGF-I 

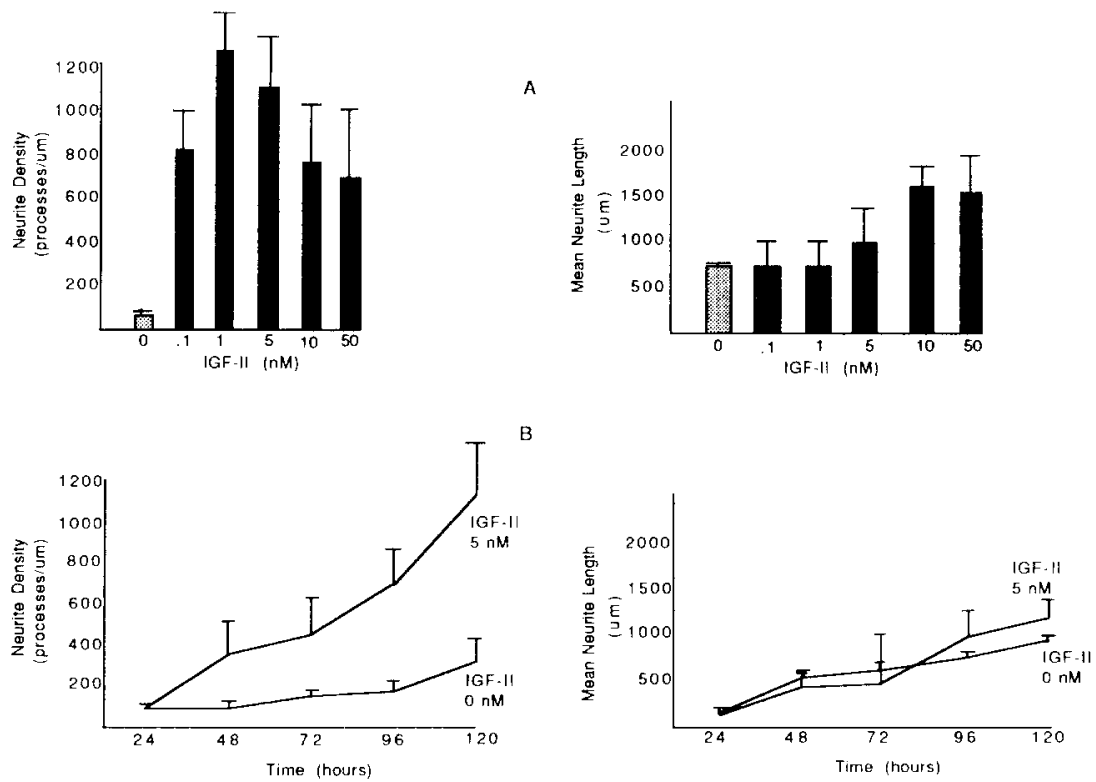

FIG 4

Measurement of neurite density and length in response to IGF II exposure. For length, measurements were significantly increased relative to control for concentrations of $10 \mathrm{nM}$ or greater. All measurements of neurite density were significantly increased compared to control (panels A).

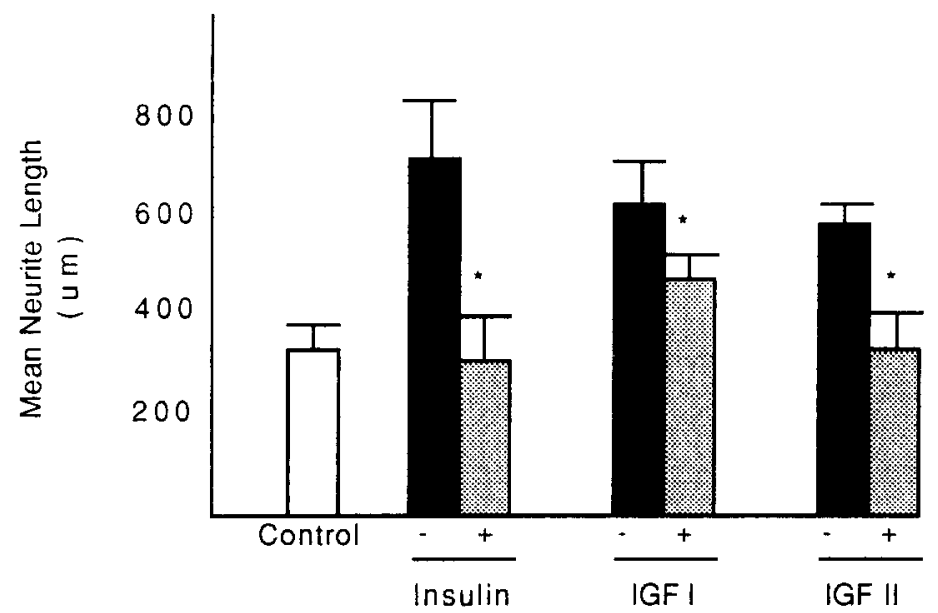

FIG 5

Mean neurite length in myenteric plexus explants exposed to insulin (2 $\mathrm{nM})$, IGF I $(5 \mathrm{nM})$, or IGF II $(5 \mathrm{nM})$ in the absence $(-)$ or presence $(+)$ of ARA-C. ${ }^{*} \mathrm{P}<0.05$ vs. trophic agonist alone. 
or IGF-II is a secondary consequence of activation of an intrinsic trophic factor. If so, non-neuronal cells may be implicated in the production, processing, or action of such a factor; ARA-C treatment would be expected to affect non-neuronal actions. Insulin or insulin-like growth factors could also act to promote neuronal survival and to allow expression in cell culture of trophic signals received in vivo. This last possibility seems unlikely given the relatively small neurite outgrowth observed in the absence of insulin or IGF in control groups over 120 hours of observation.

Determination of the physiological relevance of these observations will require additional investigation. Demonstration of cellular receptors for these peptides will be necessary to assess the specificity of the observed trophic actions. In several neuronal tissues or cell lines, demonstration of specific receptors has been possible for insulin-related peptides that exhibit neurotrophic activities $(3,5,10,17,19)$. Determination of precise structural and functional relationships that apply for enteric neurons may be hampered, however, by the heterogeneity of myenteric plexus neuronal cell types.

Accumulating evidence indicates that insulin and insulin-like growth factors may have multiple developmental functions in nervous tissues. In the central nervous system, insulin accelerates maturation of retinal neurons derived from fetal rats, as measured by increased cholinergic synaptic transmission (13). Choline acetyltransferase activity is increased in cultured fetal chicken retinal neurons exposed to insulin (7). IGF-I stimulates DNA synthesis (8) and promotes development of myelin-synthesizing oligodendrocytes in fetal rat brain cell cultures (11). In cultures derived from chick sympathetic ganglia and dorsal root ganglia, morphological development in the form of neurite formation, is stimulated by insulin and IGF-II (14). In human neuroblastoma cells, increased neurite formation is accompanied by increases in tubulin mRNA levels (12). The current results indicate that insulin and insulin-like growth factors may also have developmental effects in enteric neurons derived from neonatal guinea pigs.

The ability of insulin and related peptides to stimulate, or to sustain, morphological development in the enteric nervous system of older animals is currently unknown. Most studies have focused, instead, upon the absence of insulin in animal models of diabetes. Burnstock and co-workers have reported that specific subpopulations of enteric neurons undergo ultrastructural changes characterized by nerve fiber loss after induction of diabetes by streptozotocin $(4,9,10)$. When examined by electron microscopy, vasoactive intestinal peptide-containing neurons from the ileal myenteric plexus showed depletion of neurotransmitter vessicles (4). Similarly, when the ileum or colon from rats rendered diabetic for 8 weeks were examined, selective degeneration of nerve fibers staining for calcitonin gene-related peptide was noted in the myenteric plexus, while diabetes produced no change in the numbers of substance P-positive neurons. While these studies suggest that removal of endogenous insulin is deleterious to the enteric nervous system, the role of insulin and related peptides in preserving neuronal structural and functional integrity is speculative; this remains an important area for investigation.

Acknowledgement

Supported by NIH Grant DK41204 


\section{References}

1. Y. AIZENMAN, M.E. WEICHSEL and J. DE VELLIS. Proc. Natl. Acad. Sci. USA. 83 2263-2266 (1986).

2. Y. AIZENMAN and J. DE Vellis. Brain Res. 406 32-42 (1987).

3. L. BASSAS, F. De PABLO, M.A. LESNIAK and J. ROTH. Endocrinology 1172321 2329 (1985).

4. A. BELAI, J. LINCOLN, P. MILNER and G. BURNSTOCK. Gastroenterology 95 12341241 (1988).

5. S.K. BURGESS, S. JACOBS, P. CAUTRECASAS and N. SAHYOUN. J. Biol. Chem. 262 1618-1622 (1987).

6. K.R. JESSEN, M.J. SAFFREY and G. BURNSTOCK. Brain Res. 262 17-35 (1983).

7. J.M. KYRIAKIS, R.E. HAUSMAN and S.W.PETERSON. Proc. Natl. Acad. Sci. USA. 84 7463-7467 (1987).

8. D. LENOIR and P. HONEGGER. Dev. Brain Res. I 205-213 (1983).

9. J. LINCOLN, J.T. BOKOR and R. CROWE. Gastroenterology 86 654-661 (1984).

10. A. LOESCH, A. BELAI, J. LINCOLN and G. BURNSTOCK. Acta Neuropathol. (Berl) 70 161-168 (1986).

11. F.A. MCMORRIS, T.M. SMITH, S. DeSALVO and R.W. FURLANETTO. Proc. Natl. Acad. Sci. USA. 83 822-826 (1986).

12. J.F. MILL, M.V. CHAO and D.N. ISHII. Proc. NatI. Acad. Sci. USA. 82 7126-7130 (1985).

13. D.G. PURO and E. AGARDH. Science 225 1170-1172 (1984).

14. E. RECIO-PINTO, M.M. RECHLER and D.N. ISHII. J. Neurosci. 6 1211-1219 (1986).

15. R.G. ROSENFELD, G. CEDA and C.W. CUTLER. Endocrinology 117 2008-2016 (1985).

16. R.E. RYDEL and L.A. GREENE. Proc. Natl. Acad. Sci. USA. 85 1257-1261 (1988).

17. V.R. SARA, K. HALL and H. VON HOLST. Neurosci. Lett. 34 39-44 (1982).

18. M.A. STURM, C.A. CONOVER, H. PHAM and R.G. ROSENFELD. Endocrinology 124 388-396 (1989).

19. C.F.H. VAN SCHRAVENDIJK, E.L. HOOGHE-PETERS, J.L. VAN DEN BRANDE and D.G. PIPELEERS. Biochem. Biophys. Res. Comm. 135 228-238 (1986). 